\title{
ELECTRONIC PROPERTIES OF SEMICONDUCTORS
}

\section{Grain Boundary Related Electrical Transport in Al-rich $\mathrm{Al}_{x} \mathbf{G a}_{1-x} \mathbf{N}$ Layers Grown by Metal-Organic Chemical Vapor Deposition ${ }^{1}$}

\author{
A. Yildiz ${ }^{a, b}$, P. Taslic ${ }^{i}$ B. Sarikavak ${ }^{c}$, S. B. Lisesivdin ${ }^{c}$, M. K. Ozturk ${ }^{c}$, \\ M. Kasap ${ }^{c}$, S. Ozcelik ${ }^{c}$, and E. Ozbay ${ }^{d, e}$ \\ ${ }^{a}$ Department of Engineering Physics, Faculty of Engineering, Ankara University, 06100 Besevler, Ankara, Turkey \\ ^e-mail: yildizab@gmail.com \\ ${ }^{b}$ Department of Physics, Faculty of Science and Arts, Ahi Evran University, 40040 Kirsehir, Turkey \\ ${ }^{c}$ Department of Physics, Faculty of Science and Arts, Gazi University, Teknikokular, 06500 Ankara, Turkey \\ ${ }^{d}$ Department of Physics, Bilkent University, Bilkent, 06800 Ankara, Turkey \\ ${ }^{e}$ Department of Electrical and Electronics Engineering, Bilkent University, Bilkent, 06800 Ankara, Turkey
}

Submitted March 16, 2010; accepted for publication April 29, 2010

\begin{abstract}
Electrical transport data for Al-rich AlGaN layers grown by metal-organic chemical vapor deposition (MOCVD) are presented and analyzed in the temperature range 135-300 K. The temperature dependence of electrical conductivity indicated that conductivity in the films was controlled by potential barriers caused by carrier depletion at grain boundaries in the material. The Seto's grain boundary model provided a complete framework for understanding of the conductivity behavior. Various electrical parameters of the present samples such as grain boundary potential, donor concentration, surface trap density, and Debye screening length were extracted.
\end{abstract}

DOI: $10.1134 / \mathrm{S} 1063782611010234$

\section{INTRODUCTION}

In the recent years a considerable attention has been given to electrical properties of nitride compounds due to their significance in technology as well as in fundamental science. They have many applications for high electron mobility transistors (HEMT) and optoelectronic devices operating in the range from blue-green to ultraviolet [1]. Most of these applications are dependent on the remarkable quality of AlGaN. By the metal-organic chemical vapor deposition (MOCVD), high quality films of $\mathrm{AlGaN} / \mathrm{AlN}$ can be grown on sapphire substrates [2]. On the other hand, we should consider that epitaxial layers of nitrides always have columnar structure depending on conditions of a growth process [3]. It was reported that the electron transport properties in $\mathrm{GaN}$ were strongly influenced by grain boundaries between ordered grains in the case of columnar microcrystalline growth of $\mathrm{GaN}$ [3-5].

In order to facilitate and to improve the operation of $\mathrm{AlGaN}$ based devices, a deeper understanding of the $\mathrm{AlGaN}$ electrical properties is demanded. However, when the carrier mobility in $\mathrm{AlGaN}$ is very small, the electron transport data are limited by the conductivity measurements. Therefore, electrical conductivity is a property of fundamental interest as well as of technological importance in such a case. The electri-

\footnotetext{
${ }^{1}$ The article is published in the original.
}

cal conduction mechanism of nitrides is sensitive to the crystalline nature of the structure (single/polycrystalline) $[6,7]$. When the crystalline nature of the structure is considered, some valuable information related to the electrical properties can be obtained.

The electron transport properties of $\mathrm{AlGaN}$ single crystals were investigated in detail [8-10]. On the other hand, it is not possible to say the same things for polycrystalline $\mathrm{AlGaN}$. In order to elucidate the fundamental significance of the electron transport within the polycrystalline $\mathrm{AlGaN}$ and, hopefully, to provide usefull information about conduction in these structures, here we focus on the electrical conductivity of polycrystalline AlGaN layers. The structure of polycrystals plays an important role in the electrical properties of materials. Although, there are several works on grain boundary effects on electrical properties of GaN [3-6, 11, 12], to the best of our knowledge, data on the surface trap density of $\mathrm{AlGaN}$ layers have not been reported so far using temperature dependence of conductivity data. Therefore, it is important to determine this parameter for $\mathrm{AlGaN}$ based devices.

The main objective of this work is to highlight the related electrical properties of $\mathrm{AlGaN}$ layers having different grain sizes.

\section{EXPERIMENTAL}

The $n$-AlGaN/AlGaN/AlN structures were grown on $c$-plane [0001] $\mathrm{Al}_{2} \mathrm{O}_{3}$ substrates in a low-pressure 


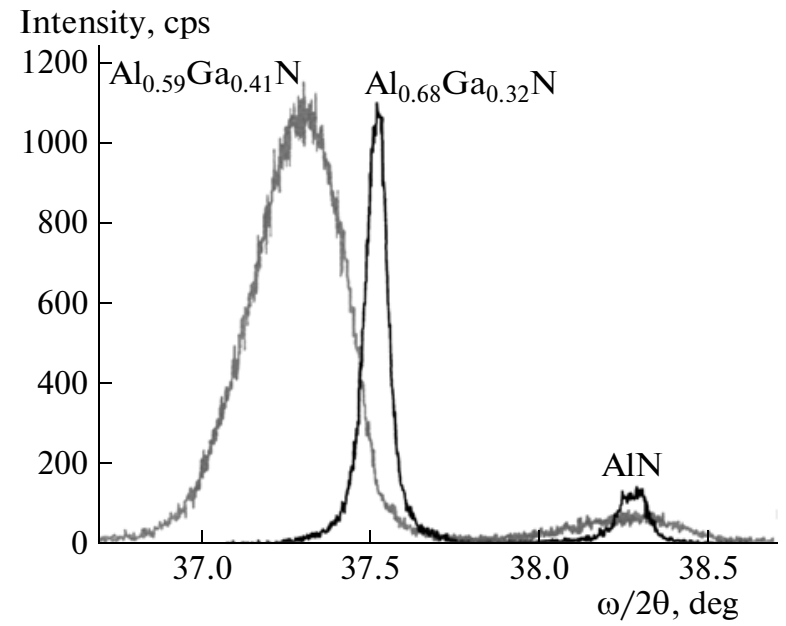

Fig. 1. HRXRD patterns of the AlGaN samples.

MOCVD reactor. Prior to epitaxial growth, the substrates were nitridated with 1000 seem $\mathrm{NH}_{3}$ flow at $790^{\circ} \mathrm{C}$. At the same temperature, thin AlN nucleation layers were grown after nitridation. Then, $\sim 150-\mathrm{nm}$ high-temperature un-doped AlN buffer layers were grown at a temperature of $1075^{\circ} \mathrm{C}$. After the AlN buffer layers, 150-nm-thick undoped AlGaN layers were grown. Lastly, 480- and 400-nm-thick Si-doped $n$-type AlGaN layers were grown for Samples $A$ and $B$, respectively. In AlGaN growth, the reactant source gas trimethylgallium (TMGa) flow was changed as $2 \mathrm{sccm}$ in Sample $B$ instead of $4 \mathrm{sccm}$ in Sample $A$. All of the layers except the last $\mathrm{AlGaN}$ layer were nominally undoped.

$\mathrm{Al}$ mole fractions in the $\mathrm{AlGaN}$ layers were determined with a simple implementation of the Bragg's law using high-resolution X-ray diffraction (HRXRD) results. HRXRD measurements were taken with D8Discover diftractometer equipped with a monochromator with four $\mathrm{Ge}(220)$ crystals for $\mathrm{Cu} K_{\alpha 1} \mathrm{X}$-ray beam (wavelength $\lambda=1.5406 \AA$ ).

Conductivity measurements were taken with a Lakeshore Hall measurement system using $5 \times 2 \mathrm{~mm}$ van der Pauw samples in the temperature range $T=$ $135-300 \mathrm{~K}$. Ohmic contacts for the conductivity measurements were prepared with evaporated triangular $\mathrm{Ti} / \mathrm{Al} / \mathrm{Ni} / \mathrm{Au}(200 / 2000 / 300 / 700 \AA)$ metals in the sample corners. After rapid thermal annealing ohmic behavior of the resulted contacts was confirmed by the current-voltage measurements.

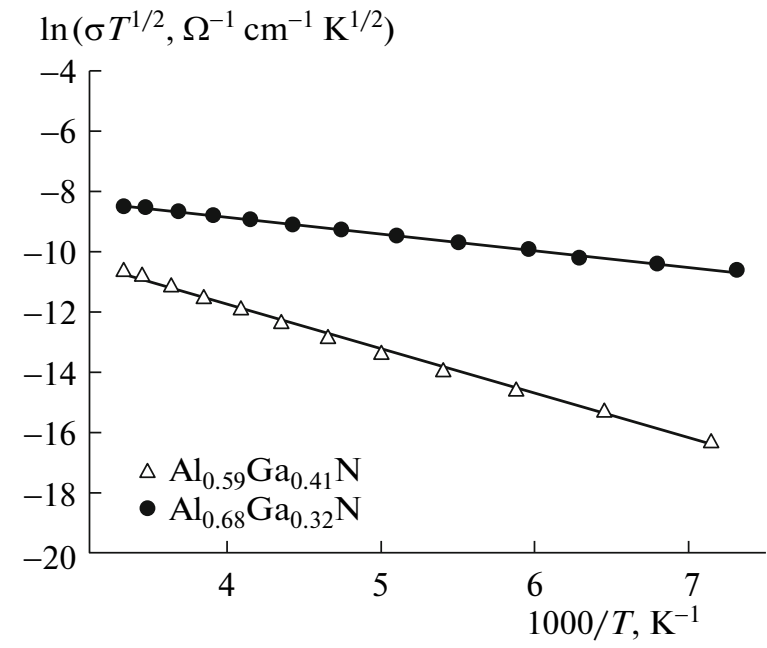

Fig. 2. Temperature dependence of the conductivity plotted as $\ln \left(\sigma T^{1 / 2}\right)$ vs. $10^{3} / T$. Solid lines are the best-fit lines with Eq. (2).

\section{RESULTS AND DISCUSSIONS}

Figure 1 shows the high resolution close-up view of the diffraction peaks obtained using HRXRD measurements for the samples. In order to determine the aluminium content $(x)$ and the grain size $(L)$ in the samples, we scanned the (0004) $\omega / 2 \theta$ reflections of hexagonal structure. From peak separations between $\mathrm{AlGaN}$ and $\mathrm{AlN}$ reflections, values of $x$ were found using LEPTOS 4.02 with dynamic theory [13]. The values of $L$ for the samples were calculated with the Debye-Scherrer formula [14]

$$
L=\frac{0.9 \lambda}{B \cos \theta}
$$

where $B$ is the peak width, $\theta$ is the diffraction angle and $\lambda$ is the $\mathrm{X}$-ray wavelength corresponding to $\mathrm{Cu} K_{\alpha}$ radiation. The values of the parameters $x$, and $L$ are collected in table. The HRXRD measurements indicate that the film with $x=0.68$ have larger crystallites than the film with $x=0.59$ (table).

Figure 2 shows the plots of $\ln \left(\sigma T^{1 / 2}\right)$ ( $\sigma$ is the conductivity) vs. $1000 / T$ for the samples investigated. These data demonstrate that the conductivity increases with increase of grain size. This can be attributed to improvement in crystalline structure and leads to improvement in the conductivity. It is in agreement with Seto's grain boundary model [15] of

Thickness $(t)$, the average grain size $(L)$, barrier height $\left(E_{b}\right)$, donor concentration $\left(N_{D}\right)$, Debay screening length $\left(L_{\mathrm{D}}\right)$, and surface trap density $\left(Q_{t}\right)$ for the AlGaN samples

\begin{tabular}{c|c|c|c|c|c|c}
\hline Sample & $t, \mathrm{~nm}$ & $L, \mathrm{~nm}$ & $E_{b}, \mathrm{meV}$ & $N_{D}, \mathrm{~cm}^{-3}$ & $L_{\mathrm{D}}, \mathrm{nm}$ & $Q_{t}, \mathrm{~cm}^{-2}$ \\
\hline $\mathrm{Al}_{0.59} \mathrm{Ga}_{0.41} \mathrm{~N}$ & 480 & 13.6 & 125 & $3.44 \times 10^{18}$ & 2.18 & $4.72 \times 10^{12}$ \\
$\mathrm{Al}_{0.68} \mathrm{Ga}_{0.32} \mathrm{~N}$ & 400 & 88 & 49 & $3.27 \times 10^{16}$ & 22.6 & $2.88 \times 10^{11}$ \\
\hline
\end{tabular}


the conductivity which showed increase of conductivity with increasing grain size. A noticeable increase in conductivity with increasing grain size is observed in our case. The conductivity of the films increased from $2.28 \times 10^{-5}$ to $2.02 \times 10^{-4}(\Omega \mathrm{cm})^{-1}$ at room temperature with the grain size increase from 13.6 to $88 \mathrm{~nm}$, respectively. This can be elucidated on the basis of the Seto's grain boundary model. Then, it may be expected that the temperature dependence of the conductivity obeys the Seto's relation [15]

$$
\sigma=\left(\frac{L e^{2} n v_{c}}{k_{\mathrm{B}} T}\right) \exp \left(-\frac{E_{b}}{k_{\mathrm{B}} T}\right)
$$

where $e$ is the electron charge, $n$ is the electron concentration in neutral region of crystallites, $k_{\mathrm{B}}$ is the Boltzmann constant, $E_{b}$ is the barrier energy at the boundary and $V_{c}$ is the collection velocity. $E_{b}$ can be described as [15]

$$
E_{b}=\frac{L^{2} e^{2} N_{D}}{8 \varepsilon},
$$

where $\varepsilon$ is the low frequency dielectric constant and $N_{D}$ is the donor concentration. $V_{c}$ is expressed as [15]

$$
V_{c}=\left(\frac{k_{\mathrm{B}} T}{2 \pi m^{*}}\right)^{1 / 2},
$$

$m^{*}$ is the effective mass of charge carriers. By using the iteration method [16], the values of $m^{*}$ and $e$ of $\mathrm{Al}_{x} \mathrm{Ga}_{1-x} \mathrm{~N}$ alloys as a function of $x$ can be evaluated Here, we used the values of effective mass $m^{*}=0.22 m_{0}$ and $0.48 m_{0}$, and the static dielectric constants $\varepsilon=10.4$ and 8.5 for GaN and AlN, respectively [16]. Applicability of the grain boundary model involves many grain boundaries. This effect is examined by evaluation of the Debye screening length $\left(L_{\mathrm{D}}\right)$ in comparison with $L . L_{\mathrm{D}}$ is given as [17]

$$
L_{\mathrm{D}}=\sqrt{k_{\mathrm{B}} T \varepsilon_{0} \varepsilon / e^{2} N_{D}},
$$

where $\varepsilon_{0}$ is the dielectric constant of vacuum. If $L_{\mathrm{D}}<$ $L / 2$, potential barriers exist in grain boundary region due to interface trap states [17]. If, however, $L_{\mathrm{D}}$ is larger than $L / 2$, the conduction band becomes flat without the potential barrier [17], and the electrons are transported without grain boundary scattering.

Since a polycrystalline film has crystallites joined at their surfaces via grain boundaries, the boundaries between crystallites play an important role in determination of conductivity of polycrystalline films. In a polycrystalline material, high densities of defects are expected at grain boundaries which are often charged with majority carriers. The charged states at grain boundaries create depleted regions which also act as potential barriers [15, 17].

If we return to Fig. 2, the symbols in Fig. 2 are the experimental data and the solid lines are the best fitted values with Eq. (2); $r^{2}=0.99$ ( $r$ is the correlation coefficient) is obtained, which indicates the satisfactory fit. The linearity of the plots reveals that the grain boundary scattering of charge carriers is more predominant in the samples investigated. The potential barrier height in the films $\left(E_{b}\right)$ and the value of donor concentration $\left(N_{D}\right)$ were calculated from the slope of the curves in Fig. 2. The potential barrier height in the films decreased with increasing grain size. Decrease of the potential barrier height is due to increase of crystallite size resulting in diminishing charge carrier scattering at the grain boundaries. The shrinkage of a grain size leads to an increment in the trapping states at a grain boundary. Trapping states are capable of trapping free carriers and, as a consequence, more free carriers become immobilized as the density of trapping states increases. In other words, the larger grain size results in the lower density of grain boundaries, which behave as traps for free carriers and barriers for carrier transport in the film. Hence, increase in the grain size can cause decrease in grain boundary scattering, which leads to increase in the conductivity.

Knowing the values of $N_{D}$, the values of the Debye screening length $\left(L_{\mathrm{D}}\right)$ can be calculated. The calculated values of $L_{\mathrm{D}}$ from Eq. (5) are given in table. Note that the condition $L_{\mathrm{D}}<L / 2$, appropriate for the grain boundary model is obeyed here for both samples. Thus, the approach of analyzing the data using the grain boundary model for thermal activation of conductivity is proper for both samples.

Charged states at the grain boundaries create the depleted regions and the potential barriers which provide resistance for the passage of carriers [15, 17]. This situation was also reported for nitrides [4-6]. Trapping states are capable to trap free carriers and, as a consequence, more free carriers become immobilized as number of trapping states increases. Now, the surface trap densities $\left(Q_{t}\right)$ in the films can be estimated using the relation [15]

$$
Q_{t}=\frac{\left(8 \varepsilon \varepsilon_{0} N_{D} E_{b}\right)^{1 / 2}}{e} .
$$

Substituting the values of $N_{D}$ and $E_{b}$ into Eq. (6), the values of $Q_{t}$ are found and they are presented in table. The value of grain boundary surface trap density $\left(Q_{r}\right)$ should agree with the value of the surface state density of various systems having the same origin. $Q_{r}$ is well in agreement with reported values for both nitride and other polycrystalline systems $[15,17,18-20]$. The values of $Q_{r}$ decrease with increasing grain size and match the experimental data as expected [15]. Decrease in $Q_{r}$ was also observed in $\mathrm{AlGaN} / \mathrm{GaN}$ heterostructures after $\mathrm{Si}_{3} \mathrm{~N}_{4}$ passivation [18]. The improvement in the alignment of the grains at the grain boundaries minimizes the trapping of charge carriers at the grain boundaries.

The surface trap density $\left(Q_{t}\right)$ in Eq. (6) depends on the relation between $N_{D}$ and $E_{b}$. It was reported that for 
higher values of $N_{D}$ in the grain inside, a higher $Q_{t}$ is needed to form the potential barrier in $\mathrm{GaN}$ [4]. Here, this situation is also confirmed for our $\mathrm{AlGaN}$ layers.

\section{CONCLUSIONS}

Electron transport data for $\mathrm{Al}$-rich $\mathrm{AlGaN}$ layers grown by metal-organic chemical vapor deposition (MOCVD) are presented and analyzed in the temperature range $135-300 \mathrm{~K}$. The data on temperature dependence of the conductivity were analyzed in terms of the grain boundary model. Characteristic grain boundary parameters, such as grain boundary potential, donor concentration, surface trap density, and Debye screening length, were all determined from our measurements where we show that their values quite well agree with the assumptions of the Seto's grain boundary model. It was found that the conductivity increases with increasing grain size. The potential barrier height and surface trap density decreased due to increase in grain size.

\section{ACKNOWLEDGMENTS}

This work is supported by the State of Planning Organization of Turkey under Grant no. 2001K120590 and European Union under the projects EU-PHOME, and EU-ECONAM, and TUBITAK under Projects $\mathrm{N}$ 106E198, 107A004, and 107A012. One of the authors (E. Ozbay) also acknowledges partial support from the Turkish Academy of Sciences.

\section{REFERENCES}

1. S. Nakamura, S. Pearton, and G. Fasol, The Blue Laser Diode: The Complete Story (Springer, New York, 2000).

2. S. B. Lisesivdin, A. Yildiz, S. Acar, M. Kasap, S. Ozcelik, and E. Ozbay, Appl. Phys. Lett. 91, 102113 (2007).
3. P. Raszkiewicz, B. Paszkiewicz, J. Kozlowski, T. Piasecki, W. Kośnikowski, and M. Tlaczala, J. Cryst. Growth 248, 487 (2003).

4. A. Szyszka, B. Paszkiewicz, R. Paszkiewicz, and M. Tlaczala, Mater. Sci. (Poland) 26, 221 (2008).

5. I. Shalish, L. Kronik, Y. Shapira, S. Zamir, B. Meyler, and J. Salzman, Phys. Rev. B 61, 15573 (2000).

6. A. Szyszka, B. Paszkiewicz, R. Paszkiewicz, and M. Tlaczala, Vacuum 82, 1034 (2008).

7. A. Yildiz, S. B. Lisesivdin, M. Kasap, S. Ozcelik, E. Ozbay, and N. Balkan, Appl. Phys. A 98, 557 (2010).

8. S. B. Lisesivdin, N. Balkan, O. Makarovsky, A. Patanè, A. Yildiz, M. D. Caliskan, M. Kasap, S. Ozcelik, and E. Ozbay, J. Appl. Phys. 105, 093701 (2009).

9. S. B. Lisesivdin, S. Demirezen, M. D. Caliskan, A. Yildiz, M. Kasap, S. Ozcelik, and E. Ozbay, Semicond. Sci. Technol. 23, 095008 (2008).

10. S. Acar, S. B. Lisesivdin, M. Kasap, S. Ozcelik, and E. Ozbay, Thin Sol. Films 516, 2041 (2008).

11. J. Oila, K. Saarinen, A. E. Wickenden, D. D. Koleske, R. L. Henry, and M. E. Twigg, Appl. Phys. Lett. 82, 1021 (2003).

12. N. Sarkar, S. Dhar, and S. Ghosh, J. Phys.: Condens. Matter 15, 7325 (2003).

13. Diffrac Plus 2006. TOPAS v. 3.0, The Manual (BRUKER AXS GmbH, Karlsruhe, 2006).

14. B. D. Cullity, Elements of X-Ray Diffraction, 2nd ed. (Addison-Wesley, Reading, MA, 1978).

15. J. Y. W. Seto, J. Appl. Phys. 46, 5247 (1975).

16. H. Morkoc, Nitride Semiconductors and Devices (Springer, Heidelberg, 1999).

17. J. W. Orton and M. J. Powel, Rep. Progr. Phys. 43, 1263 (1980).

18. J. Bernát, P. Javorka, M. Marso, and P. Kordoš, Appl. Phys. Lett. 83, 5455 (2003).

19. J. Dutta, D. Bhattacharyya, A. B. Maiti, and A. K. Pal, Vacuum 46, 17 (1995).

20. J. Salzman, C. Uzan-Saguy, B. Meyler, and R. Kalish, Phys. Status Solidi A 176, 683 (1999). 